\title{
An Investigation on the Beta Function IV: An approximation of the Error Function
}

\section{Edigles Guedes ${ }^{1}$ and Prof. Dr. K. Raja Rama Gandhi ${ }^{2}$}

Number Theorist, Brazil ${ }^{1}$

Resource perosn in Mathematics for Oxford University Press and Professor at BITS-Vizag ${ }^{2}$

Abstract. I discovered an approximation of the error function.

\section{INTRODUCTION}

In this paper, I discovered an approximation of the error function:

$$
\operatorname{erf}(z) \cong \frac{8 z \tan ^{-1}(z)-4 \ln \left(z^{2}+1\right)-2 z^{2}}{z \sqrt{\pi}}
$$

for $0<z \leq 1$.

\section{THEOREMS}

Theorem 1. For $\mathfrak{R}(x)>0$ and $\mathfrak{R}(y)>0$, then

$$
\frac{\Gamma(x) \Gamma(y) \Gamma\left(x+y+\frac{1}{2}\right)}{\Gamma(x+y) \Gamma\left(y+\frac{1}{2}\right)}=\int_{0}^{\infty} e^{-t}{ }_{1} F_{1}\left(\frac{1}{2} ; x+y+\frac{1}{2} ; t\right) t^{x-1} \mathrm{~d} t,
$$

where ${ }_{1} F_{1}(a ; b ; z)$ denotes the hypergeometric function.

Proof. In [1, page 883], I have the Euler's definition of gamma function

$$
\Gamma(z) \stackrel{\text { def }}{=} \int_{0}^{\infty} e^{-t} t^{z-1} \mathrm{~d} t
$$

for $\mathfrak{R}(x)>0$. I substitute (1) into Theorem 1 of previous paper [2]

$$
\begin{gathered}
G_{2}(x, y)=\sum_{k=0}^{\infty} \frac{(2 k) !}{4^{k} k !^{2} \Gamma\left(x+y+k+\frac{1}{2}\right)} \int_{0}^{\infty} e^{-t} t^{x+k-1} \mathrm{~d} t \\
=\int_{0}^{\infty} e^{-t} \sum_{k=0}^{\infty} \frac{(2 k) ! t^{k}}{4^{k} k !^{2} \Gamma\left(x+y+k+\frac{1}{2}\right)} t^{x-1} \mathrm{~d} t \\
=\int_{0}^{\infty} e^{-t} \frac{{ }_{1} F_{1}\left(\frac{1}{2} ; x+y+\frac{1}{2} ; t\right)}{\Gamma\left(x+y+\frac{1}{2}\right)} t^{x-1} \mathrm{~d} t \\
=\frac{1}{\Gamma\left(x+y+\frac{1}{2}\right)} \int_{0}^{\infty} e^{-t}{ }_{1} F_{1}\left(\frac{1}{2} ; x+y+\frac{1}{2} ; t\right) t^{x-1} \mathrm{~d} t
\end{gathered}
$$

so,

$$
\Gamma\left(x+y+\frac{1}{2}\right) G_{2}(x, y)=\int_{0}^{\infty} e^{-t}{ }_{1} F_{1}\left(\frac{1}{2} ; x+y+\frac{1}{2} ; t\right) t^{x-1} \mathrm{~d} t
$$


in other words,

$$
\frac{\Gamma(x) \Gamma(y) \Gamma\left(x+y+\frac{1}{2}\right)}{\Gamma(x+y) \Gamma\left(y+\frac{1}{2}\right)}=\int_{0}^{\infty} e^{-t}{ }_{1} F_{1}\left(\frac{1}{2} ; x+y+\frac{1}{2} ; t\right) t^{x-1} \mathrm{~d} t . \square
$$

Theorem 2. For $\mathfrak{R}(x)>0$ and $\mathfrak{R}(y)>0$, then

$$
\frac{\Gamma(x) \Gamma(y) \Gamma\left(x+y+\frac{1}{2}\right)}{\Gamma(x+y) \Gamma\left(y+\frac{1}{2}\right)}=\sum_{n=0}^{\infty} \frac{\left(\frac{1}{2}\right)_{n} \Gamma(x+n)}{\left(x+y+\frac{1}{2}\right)_{n} n !} .
$$

Proof. By Theorem 1, I have

$$
\begin{gathered}
\frac{\Gamma(x) \Gamma(y) \Gamma\left(x+y+\frac{1}{2}\right)}{\Gamma(x+y) \Gamma\left(y+\frac{1}{2}\right)}=\int_{0}^{\infty} e^{-t} \sum_{n=0}^{\infty} \frac{\left(\frac{1}{2}\right)_{n} t^{n}}{\left(x+y+\frac{1}{2}\right)_{n} n !} t^{x-1} \mathrm{~d} t \\
=\sum_{n=0}^{\infty} \frac{\left(\frac{1}{2}\right)_{n}}{\left(x+y+\frac{1}{2}\right)_{n} n !} \int_{0}^{\infty} e^{-t} t^{x+n-1} \mathrm{~d} t \\
=\sum_{n=0}^{\infty} \frac{\left(\frac{1}{2}\right)_{n} \Gamma(x+n)}{\left(x+y+\frac{1}{2}\right)_{n} n !}
\end{gathered}
$$

Corollary 1. Let $n$ and $k$ are positive integers and $n>k$, then

$$
\left(\begin{array}{l}
n \\
k
\end{array}\right)=\frac{\Gamma\left(n+k+\frac{3}{2}\right)}{\Gamma(n-k+1) \Gamma\left(k+\frac{1}{2}\right) \Gamma(n+k+2)} \sum_{r=0}^{\infty} \frac{\left(\frac{1}{2}\right)_{r} \Gamma(n+r+1)}{(n+k+2)_{r} r !} .
$$

Proof. Let $x \rightarrow x+1$ and $y \rightarrow y+\frac{1}{2}$ in Theorem 2

$$
\frac{\Gamma(x+1) \Gamma\left(y+\frac{1}{2}\right) \Gamma(x+y+2)}{\Gamma\left(x+y+\frac{3}{2}\right) \Gamma(y+1)}=\sum_{r=0}^{\infty} \frac{\left(\frac{1}{2}\right)_{r} \Gamma(x+r+1)}{(x+y+2)_{r} r !}
$$

Multiply by $\frac{1}{\Gamma(x-y+1)}$

$$
\begin{gathered}
\frac{\Gamma(x+1) \Gamma\left(y+\frac{1}{2}\right) \Gamma(x+y+2)}{\Gamma(x-y+1) \Gamma\left(x+y+\frac{3}{2}\right) \Gamma(y+1)}=\frac{1}{\Gamma(x-y+1)} \sum_{r=0}^{\infty} \frac{\left(\frac{1}{2}\right)_{r} \Gamma(x+r+1)}{(x+y+2)_{r} r !} \Rightarrow \\
\frac{\Gamma(x+1)}{\Gamma(x-y+1) \Gamma(y+1)}=\frac{\Gamma\left(x+y+\frac{3}{2}\right)}{\Gamma(x-y+1) \Gamma\left(y+\frac{1}{2}\right) \Gamma(x+y+2)} \sum_{r=0}^{\infty} \frac{\left(\frac{1}{2}\right)_{r} \Gamma(x+r+1)}{(x+y+2)_{r} r !} \Rightarrow
\end{gathered}
$$




$$
\left(\begin{array}{l}
x \\
y
\end{array}\right)=\frac{\Gamma\left(x+y+\frac{3}{2}\right)}{\Gamma(x-y+1) \Gamma\left(y+\frac{1}{2}\right) \Gamma(x+y+2)} \sum_{r=0}^{\infty} \frac{\left(\frac{1}{2}\right)_{r} \Gamma(x+r+1)}{(x+y+2)_{r} r !} .
$$

Letting $x=n$ and $y=k$, I complete the proof.

Theorem 3. For $0>z$, then

$$
\begin{aligned}
& \frac{\sqrt{\pi} \operatorname{erfi}(z)}{2 z}=\frac{z^{2 l+2}}{(2 l+3) \Gamma(l+2)}{ }_{2} F_{2}\left(\begin{array}{c}
1, l+\frac{3}{2} \\
l+2, l+\frac{5}{2}
\end{array} \mid z^{2}\right) \\
& +\sum_{r=0}^{\infty} \frac{\left(\frac{1}{2}\right)_{r}}{r !}\left[\sum_{n=0}^{l} \frac{\Gamma\left(3 n+\frac{3}{2}\right) \Gamma(2 n+r+1)}{\Gamma(n+1) \Gamma\left(n+\frac{1}{2}\right) \Gamma(3 n+2)(3 n+2)_{r}\left(\frac{3}{2}\right)_{n} 2^{2 n}}\right] \text {, }
\end{aligned}
$$

where erfi $(x)$ denotes the imaginary error function and ${ }_{2} F_{2}\left(\begin{array}{l}a, b \\ c, d\end{array} \mid z\right)$ denotes the hypergeometric function.

Proof. Put $n \rightarrow 2 n$ and $k \rightarrow n$ in Corollary 1

$$
\left(\begin{array}{c}
2 n \\
n
\end{array}\right)=\frac{\Gamma\left(3 n+\frac{3}{2}\right)}{\Gamma(n+1) \Gamma\left(n+\frac{1}{2}\right) \Gamma(3 n+2)} \sum_{r=0}^{\infty} \frac{\left(\frac{1}{2}\right)_{r} \Gamma(2 n+r+1)}{(3 n+2)_{r} r !} .
$$

Multiply by $\frac{x^{2 n}}{\left(\frac{3}{2}\right)_{n} 2^{2 n}}$ and sum from 0 at $l$ in $n$, then

$$
\begin{gathered}
\sum_{n=0}^{l}\left(\begin{array}{c}
2 n \\
n
\end{array}\right) \frac{x^{2 n}}{\left(\frac{3}{2}\right)_{n} 2^{2 n}}=\sum_{n=0}^{l} \frac{\Gamma\left(3 n+\frac{3}{2}\right)}{\Gamma(n+1) \Gamma\left(n+\frac{1}{2}\right) \Gamma(3 n+2)} \sum_{r=0}^{\infty} \frac{\left(\frac{1}{2}\right)_{r} \Gamma(2 n+r+1)}{(3 n+2)_{r} r !} \frac{x^{2 n}}{\left(\frac{3}{2}\right)_{n} 2^{2 n}} \\
=\sum_{r=0}^{\infty} \frac{\left(\frac{1}{2}\right)_{r}}{r !} \sum_{n=0}^{l} \frac{\Gamma\left(3 n+\frac{3}{2}\right) \Gamma(2 n+r+1)}{\Gamma(n+1) \Gamma\left(n+\frac{1}{2}\right) \Gamma(3 n+2)(3 n+2)_{r}} \frac{x^{2 n}}{\left(\frac{3}{2}\right)_{n} 2^{2 n}}
\end{gathered}
$$

I calculate the left hand side of (3)

$$
\sum_{n=0}^{l}\left(\begin{array}{c}
2 n \\
n
\end{array}\right) \frac{x^{2 n}}{\left(\frac{3}{2}\right)_{n} 2^{2 n}}=\frac{\sqrt{\pi} \operatorname{erfi}(x)}{2 x}-\frac{x^{2 l+2}}{(2 l+3) \Gamma(l+2)}{ }_{2} F_{2}\left(\begin{array}{c}
1, l+\frac{3}{2} \\
l+2, l+\frac{5}{2}
\end{array} \mid x^{2}\right) .
$$

I substitute (4) in the left hand side of (3), put $z$ by $x$ and after of some algebraic manipulation the proof this complete.

Corollary 2. For $z>0$, then

$$
\frac{\sqrt{\pi} \operatorname{erfi}(z)}{2 z}=1+\frac{z^{2}}{3}{ }_{2} F_{2}\left(\begin{array}{r}
1, \frac{3}{2} \\
2, \frac{5}{2}
\end{array} \mid z^{2}\right)
$$

where erfi $(x)$ denotes the imaginary error function and ${ }_{2} F_{2}\left(\begin{array}{l}a, b \\ c, d\end{array} \mid z\right)$ denotes the hypergeometric function. 
Proof. I replace 0 by $l$ in Theorem 3 .

Corollary 3. For $z>0$, then

$$
\frac{\sqrt{\pi} \operatorname{erfi}(z)}{2 z}=1+\frac{z^{2}}{3}+\frac{z^{4}}{10}{ }_{2} F_{2}\left(\begin{array}{r}
1, \frac{5}{2} \\
7, \frac{7}{2}
\end{array} \mid z^{2}\right)
$$

where erfi $(x)$ denotes the imaginary error function and ${ }_{2} F_{2}\left(\begin{array}{l}a, b \\ c, d\end{array} \mid z\right)$ denotes the hypergeometric function.

Proof. I replace 1 by $l$ in Theorem 3 .

Theorem 4. For $0<z \leq 1$, then

$$
\operatorname{erf}(z) \cong \frac{8 z \tan ^{-1}(z)-4 \ln \left(z^{2}+1\right)-2 z^{2}}{z \sqrt{\pi}}
$$

where $\operatorname{erf}(x)$ denotes the error function $\tan ^{-1}(z)$ denotes the inverse tangent function and $\ln (z)$ denotes the natural logarithm function.

Proof. Put $x \rightarrow n+1$ and $y \rightarrow 1$ in Theorem 2

$$
\frac{1}{n !}=\frac{(n+1) \sqrt{\pi}}{2 \Gamma(n+1) \Gamma\left(n+\frac{5}{2}\right)} \sum_{r=0}^{\infty} \frac{\left(\frac{1}{2}\right)_{r} \Gamma(n+r+1)}{\left(n+\frac{5}{2}\right)_{r} r !}
$$

Multiply by $\frac{2(-1)^{n} z^{2 n+1}}{\sqrt{\pi}(2 n+1)}$ and sum from 0 at $\infty$ in $n$, then

$$
\begin{gathered}
\operatorname{erf}(z)=\sum_{n=0}^{\infty} \frac{(-1)^{n} z^{2 n+1}(n+1)}{(2 n+1) \Gamma(n+1) \Gamma\left(n+\frac{5}{2}\right)} \sum_{r=0}^{\infty} \frac{\left(\frac{1}{2}\right)_{r} \Gamma(n+r+1)}{\left(n+\frac{5}{2}\right)_{r} r !} \\
=\sum_{n=0}^{\infty} \frac{(-1)^{n} z^{2 n+1} n}{(2 n+1) \Gamma(n+1) \Gamma\left(n+\frac{5}{2}\right)} \sum_{r=0}^{\infty} \frac{\left(\frac{1}{2}\right)_{r} \Gamma(n+r+1)}{\left(n+\frac{5}{2}\right)_{r} r !}+\sum_{n=0}^{\infty} \frac{(-1)^{n} z^{2 n+1}}{(2 n+1) \Gamma(n+1) \Gamma\left(n+\frac{5}{2}\right)} \sum_{r=0}^{\infty} \frac{\left(\frac{1}{2}\right)_{r} \Gamma(n+r+1)}{\left(n+\frac{5}{2}\right)_{r} r !},
\end{gathered}
$$

since $0<\mathrm{z} \leq 1$, I have

$$
\begin{gathered}
\cong \sum_{r=0}^{\infty} \frac{\left(\frac{1}{2}\right)_{r}}{r !} \sum_{n=0}^{\infty} \frac{(-1)^{n} n \Gamma(n+r+1) z^{2 n+1}}{(2 n+1) \Gamma(n+1) \Gamma\left(n+\frac{5}{2}\right)\left(n+\frac{5}{2}\right)_{r}}+\sum_{r=0}^{\infty} \frac{\left(\frac{1}{2}\right)_{r}}{r !} \sum_{n=0}^{\infty} \frac{(-1)^{n} \Gamma(n+r+1) z^{2 n+1}}{(2 n+1) \Gamma(n+1) \Gamma\left(n+\frac{5}{2}\right)\left(n+\frac{5}{2}\right)_{r}} \\
\left.=-\frac{8 z^{3}}{45 \sqrt{\pi}} \sum_{r=0}^{\infty} \frac{(r+1) !\left(\frac{1}{2}\right)_{r}}{r !\left(\frac{7}{2}\right)_{r}} F_{2}\left(\frac{\frac{3}{2}, r+2}{\frac{5}{2}, r+\frac{7}{2}}\right)^{2}\right)+\frac{4 z}{3 \sqrt{\pi}} \sum_{r=0}^{\infty} \frac{\left(\frac{1}{2}\right)_{r}}{\left(\frac{5}{2}\right)_{r}} F_{2}\left(\frac{1}{2}, r+1\right. \\
\left.\left.\frac{3}{2}, r+\frac{5}{2}\right)-z^{2}\right) \\
=-\frac{8 z^{3}}{45 \sqrt{\pi}} \sum_{r=0}^{\infty} \frac{(r+1) !\left(\frac{1}{2}\right)_{r}}{r !\left(\frac{7}{2}\right)_{r}} \sum_{n=0}^{\infty}(-1)^{n} \frac{\left(\frac{3}{2}\right)_{n}(r+2)_{n}}{\left(\frac{5}{2}\right)_{n}\left(r+\frac{7}{2}\right)_{n}} z^{2 n}+\frac{4 z}{3 \sqrt{\pi}} \sum_{r=0}^{\infty} \frac{\left(\frac{1}{2}\right)_{r}}{\left(\frac{5}{2}\right)_{r}} \sum_{n=0}^{\infty}(-1)^{n} \frac{\left(\frac{1}{2}\right)_{n}(r+1)_{n}}{\left(\frac{3}{2}\right)_{n}\left(r+\frac{5}{2}\right)_{n}} z^{2 n}
\end{gathered}
$$




$$
\begin{aligned}
& =-\frac{8 z^{3}}{45 \sqrt{\pi}} \sum_{n=0}^{\infty}(-1)^{n} \frac{\left(\frac{3}{2}\right)_{n}}{\left(\frac{5}{2}\right)_{n}} \sum_{r=0}^{\infty} \frac{(r+1) !\left(\frac{1}{2}\right)_{r}(r+2)_{n}}{r !\left(\frac{7}{2}\right)_{r}\left(r+\frac{7}{2}\right)_{n}} z^{2 n}+\frac{4 z}{3 \sqrt{\pi}} \sum_{n=0}^{\infty}(-1)^{n} \frac{\left(\frac{1}{2}\right)_{n}}{\left(\frac{3}{2}\right)_{n}} \sum_{r=0}^{\infty} \frac{\left(\frac{1}{2}\right)_{r}(r+1)_{n}}{\left(\frac{5}{2}\right)_{r}\left(r+\frac{5}{2}\right)_{n}} z^{2 n} \\
& =-\frac{16 z^{3}}{45 \pi} \sum_{n=0}^{\infty}(-1)^{n} \frac{\left(\frac{3}{2}\right)_{n}(2)_{n}}{\left(\frac{5}{2}\right)_{n}\left(\frac{7}{2}\right)_{n}} \frac{\Gamma\left(\frac{2 n+7}{2}\right)}{\Gamma(n+3)} z^{2 n}+\frac{8 z}{3 \pi} \sum_{n=0}^{\infty}(-1)^{n} \frac{\left(\frac{1}{2}\right)_{n}(1)_{n}}{\left(\frac{3}{2}\right)_{n}\left(\frac{5}{2}\right)_{n}} \frac{\Gamma\left(\frac{2 n+5}{2}\right)}{\Gamma(n+2)} z^{2 n} \\
& =\frac{2\left(2 z \tan ^{-1}(z)-\ln \left(z^{2}+1\right)-z^{2}\right)}{z \sqrt{\pi}}+\frac{2\left(2 z \tan ^{-1}(z)-\ln \left(z^{2}+1\right)\right)}{z \sqrt{\pi}} \\
& =\frac{4 z \tan ^{-1}(z)-2 \ln \left(z^{2}+1\right)-2 z^{2}+4 z \tan ^{-1}(z)-2 \ln \left(z^{2}+1\right)}{z \sqrt{\pi}} \\
& =\frac{8 z \tan ^{-1}(z)-4 \ln \left(z^{2}+1\right)-2 z^{2}}{z \sqrt{\pi}} . \square
\end{aligned}
$$

Special Values. Let $z=1$ in Theorem 4 , then

$$
\operatorname{erf}(1) \cong \frac{2 \pi-2-4 \ln (2)}{\sqrt{\pi}}
$$

with one place decimal correct.

Let $z=\frac{1}{2}$ in Theorem 4 , then

$$
\operatorname{erf}\left(\frac{1}{2}\right) \cong \frac{8 \tan ^{-1}\left(\frac{1}{2}\right)-1-8 \ln \left(\frac{5}{4}\right)}{\sqrt{\pi}}
$$

with two place decimal correct.

Let $z=\frac{1}{3}$ in Theorem 4 , then

$$
\operatorname{erf}\left(\frac{1}{3}\right) \cong \frac{8 \tan ^{-1}\left(\frac{1}{3}\right)-\frac{2}{3}-8 \ln \left(\frac{10}{9}\right)}{\sqrt{\pi}}
$$

with three place decimal correct.

Let $z=\frac{1}{4}$ in Theorem 4 , then

$$
\operatorname{erf}\left(\frac{1}{4}\right) \cong \frac{16 \tan ^{-1}\left(\frac{1}{4}\right)-1-32 \ln \left(\frac{17}{16}\right)}{\sqrt{\pi}}
$$

with four place decimal correct; and so on. In Appendix, I leave to the reader the Figure 1.

Note. I leave to reader: Prove that, for $0<z \leq 1$, then

$$
\frac{1}{2}+\frac{\sqrt{\pi} \operatorname{erf}(z)}{4 z} \cong \sum_{k=0}^{\infty} \frac{(-1)^{k} z^{2 k}}{(2 k+1)(k+1)}
$$

where $\operatorname{erf}(x)$ denotes the error function $\tan ^{-1}(\mathrm{z})$ denotes the inverse tangent function and $\ln (\mathrm{z})$ denotes the natural logarithm function. 
Theorem 5. For $0>z$, then

$$
\begin{aligned}
\operatorname{erf}(z)=\sum_{r=0}^{\infty} \frac{\left(\frac{1}{2}\right)_{r}}{r !} \sum_{n=0}^{l} \frac{(-1)^{n}(n+1) \Gamma(n+r+1) z^{2 n+1}}{(2 n+1) \Gamma(n+1) \Gamma\left(n+\frac{5}{2}\right)\left(n+\frac{5}{2}\right)_{r}} \\
\left.-\frac{2(-1)^{l} z^{2 l+3}}{\sqrt{\pi}(2 l+3) \Gamma(l+2)}{ }_{2} F_{2}\left(\begin{array}{c}
1, l+\frac{3}{2} \\
l+2, l+\frac{5}{2}
\end{array}\right)-z^{2}\right)
\end{aligned}
$$

where $\operatorname{erf}(z)$ denotes the error function and ${ }_{2} F_{2}\left(\begin{array}{l}a, b \\ c, d\end{array} \mid z\right)$ denotes the hypergeometric function.

Proof. Put $x \rightarrow n+1$ and $y \rightarrow 1$ in Theorem 2

$$
\frac{1}{n !}=\frac{(n+1) \sqrt{\pi}}{2 \Gamma(n+1) \Gamma\left(n+\frac{5}{2}\right)} \sum_{r=0}^{\infty} \frac{\left(\frac{1}{2}\right)_{r} \Gamma(n+r+1)}{\left(n+\frac{5}{2}\right)_{r} r !} .
$$

Multiply by $\frac{2(-1)^{n} x^{2 n+1}}{\sqrt{\pi}(2 n+1)}$ and sum from 0 at $l$ in $n$, then

$$
\begin{gathered}
\frac{2}{\sqrt{\pi}} \sum_{n=0}^{l} \frac{(-1)^{n} x^{2 n+1}}{(2 n+1) n !}=\sum_{n=0}^{l} \frac{(-1)^{n} x^{2 n+1}(n+1)}{(2 n+1) \Gamma(n+1) \Gamma\left(n+\frac{5}{2}\right)} \sum_{r=0}^{\infty} \frac{\left(\frac{1}{2}\right)_{r} \Gamma(n+r+1)}{\left(n+\frac{5}{2}\right)_{r} r !} \\
=\sum_{r=0}^{\infty} \frac{\left(\frac{1}{2}\right)_{r}}{r !} \sum_{n=0}^{l} \frac{(-1)^{n} x^{2 n+1}(n+1) \Gamma(n+r+1)}{(2 n+1) \Gamma(n+1) \Gamma\left(n+\frac{5}{2}\right)\left(n+\frac{5}{2}\right)_{r}}
\end{gathered}
$$

I calculate the left hand side of (8)

$$
\frac{2}{\sqrt{\pi}} \sum_{n=0}^{l} \frac{(-1)^{n} x^{2 n+1}}{(2 n+1) n !}=\operatorname{erf}(x)+\frac{2(-1)^{l} x^{2 l+3}}{\sqrt{\pi}(2 l+3) \Gamma(l+2)}{ }_{2} F_{2}\left(\begin{array}{c}
1, l+\frac{3}{2} \\
l+2, l+\frac{5}{2}
\end{array} \mid-x^{2}\right) .
$$

I substitute (9) in the left hand side of (8), put $z$ by $x$ and after of some algebraic manipulation the proof this complete.

Corollary 4. For $z>0$, then

$$
\operatorname{erf}(z)=\frac{2 z}{\sqrt{\pi}}-\frac{2 z^{3}}{3 \sqrt{\pi}}{ }_{2} F_{2}\left(\begin{array}{r}
1, \frac{3}{2} \\
2, \frac{5}{2}
\end{array} \mid-z^{2}\right)
$$

where $\operatorname{erf}(z)$ denotes the error function and ${ }_{2} F_{2}\left(\begin{array}{l}a, b \\ c, d\end{array} \mid z\right)$ denotes the hypergeometric function.

Proof. I replace 0 by $l$ in Theorem 4 .

Corollary 5. For $z>0$, then 


$$
\operatorname{erf}(z)=\frac{2 z}{\sqrt{\pi}}-\frac{2 z^{3}}{3 \sqrt{\pi}}+\frac{z^{5}}{5 \sqrt{\pi}}{ }_{2} F_{2}\left(\begin{array}{r}
1, \frac{5}{2} \\
3, \frac{7}{2}
\end{array} \mid-z^{2}\right)
$$

where $\operatorname{erf}(z)$ denotes the error function and ${ }_{2} F_{2}\left(\begin{array}{c}a, b \\ c, d\end{array} \mid z\right)$ denotes the hypergeometric function. Proof. I replace 1 by $l$ in Theorem 4.

\section{APPENDIX}

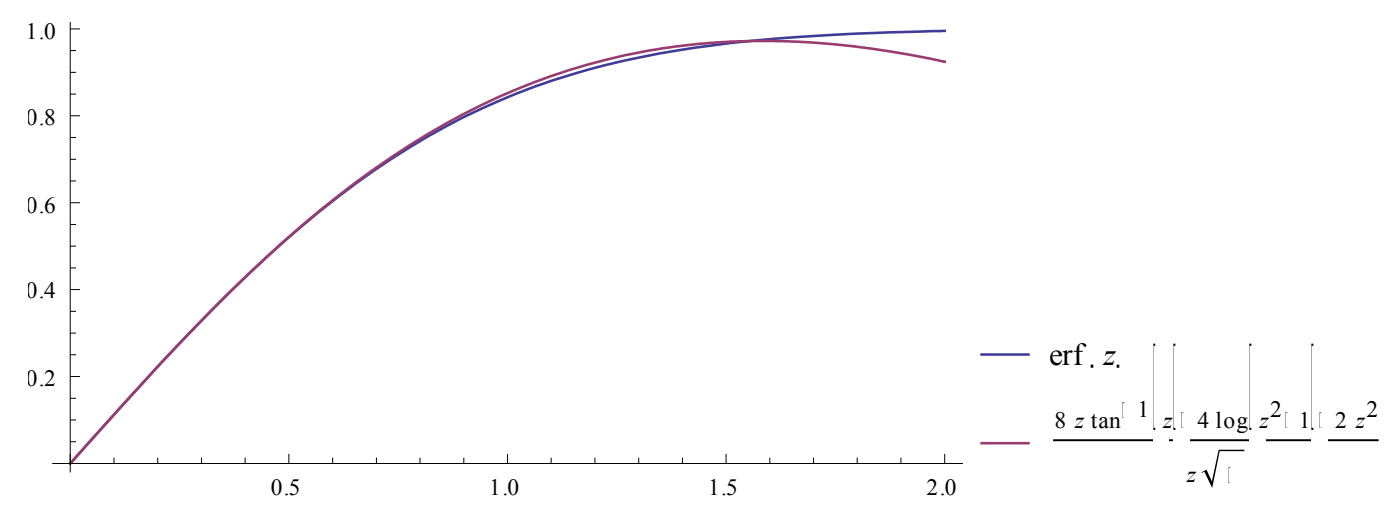

Figure 1

\section{REFERENCES}

[1] Gradshteyn, I. S. and Ryzhik, I. M., Table of Integrals, Series and Products, Academic Press, 2000 .

[2] Guedes, Edigles, An Investigation on the Beta Function I: New Versions of the Euler Beta Function, 2013. 